\title{
Effect of Orthodontic Treatment on Tooth Autotransplantation: Systematic Review of Controlled Clinical Trials
}

\author{
Rogério Lacerda-Santos ${ }^{1}$ Rhaíssa Ferreira Canutto ${ }^{1}$ José Lucas dos Santos Araújo ${ }^{2}$ \\ Fabiola Galbiatti de Carvalho ${ }^{1}$ Eliseu Aldrighi Münchow ${ }^{3}$ Taís de Souza Barbosa ${ }^{1}$ \\ Matheus Melo Pithon ${ }^{4}$ Emanuel Braga Rego ${ }^{5}$ Leniana Santos Neves ${ }^{6}$
}



\begin{abstract}
Address for correspondence Rogério Lacerda-Santos, DDS, MSD, PhD, Department of Orthodontics, Federal University of Juiz de Fora -UFJF, Faculty of Dentistry, Avenue Doutor Raimundo Monteiro Rezende, n.330, Centro, CEP:35010-177, Governador Valadares, Minas Gerais, Brazil (e-mail: lacerdaorto@hotmail.com; lacerdaorto@gmail.com).
\end{abstract}

Eur J Dent:2020;14:467-482

\begin{abstract}
This systematic review was focused on evaluating tooth autotransplantation, considering its impacts on the teeth, bone, soft tissues, and aesthetics in orthodontic patients. A bibliographic search was conducted without limitations on year of publication or language in the databases of PubMed, Web of Science, Scopus, Medline Complete, Cochrane, Clinical Trials, and Trials Central. For triage of articles, indications, surgical planning, orthodontic movement, risk factors for treatment, and long-term follow-ups were considered. For outcomes, the results with reference to teeth, alveolar bone, periodontal tissues, and esthetic satisfaction were considered. Risk of bias was evaluated using the methodological index for nonrandomized studies-MINORS. The results showed 10 controlled clinical trials, and no randomized clinical trials were found. The selected studies included 715 patients and 934 autotransplanted teeth among which there were premolars, molars, and anterior teeth evaluated in the long term, indicating that orthodontics associated with autotransplantation indicated a result that was generally clinically acceptable. The quality of the set of evidence was considered medium due to the presence

Keywords

- transplantation

- success rate

- root resorption

- survival rate

- orthodontics of different methodological problems, risk of bias, and significant heterogeneity in the evaluated studies. There was a sufficient body of evidence that justified autotransplantation in patients who needed orthodontic movement. In teeth, there was an increase in root resorption influenced by orthodontics, but without impacting on the general clinical result in the long term. Bone and periodontal tissue do not appear to be affected by orthodontics. The patient's aesthetic satisfaction was not considered in the studies.
\end{abstract}




\section{Introduction}

Autotransplantation is a valuable alternative to extraction, for replacing agenesis and/or missing teeth in rehabilitation and orthodontic treatments. ${ }^{1}$ An ectopic position or sharp angulation in relation to the root parallelism of the teeth are used criteria for selection of an autotransplantation. ${ }^{2}$ Orthodontic treatment with space closure or associated with dental implants is not always accepted by patients, due to longer treatment time involved, localization of missing teeth, and ethical and economic considerations. ${ }^{3}$ In these situations, and in the presence of sufficient diastema, tooth autotransplantation may be a suitable clinical choice.

Previous studies have described high survival rates of transplanted teeth with complete ${ }^{4-6}$ root formation and with incomplete rhizogenesis, and have shown few complications with obtaining sufficient final root length. ${ }^{7}$

Up to now, no systematic review evaluating the influence and long-term results of autotransplantation in orthodontic patients has been reported in the literature. So, the focus of this study was to conduct a systematic review about tooth autotransplantation, considering its impacts on the teeth, bone, soft tissues, and aesthetics in orthodontic patients.

\section{Materials and Methods}

\section{Focus Question}

This systematic analysis was realized to answer the focus question: Is there any scientific evidence of the reduction in survival and success rate scores of tooth autotransplantation in orthodontic patients who were submitted to movement of these teeth in comparison with a control group?

\section{Search Strategy}

The methodology applied in this systematic analysis was based on the PRISMA guidelines (Preferred Reporting Items for Systematic Review and Meta-Analysis-PRISMA) ${ }^{8}$ (accessed in: www.prisma-statement.org).
Table 1 Database and search method

\begin{tabular}{|l|l|}
\hline Database & Search strategy \\
\hline PubMed & ((orthodontics [MeSH Terms] OR orthodontic \\
Web of Science & OR orthodontic patients OR orthodontic \\
Medline & treatment OR tooth movement OR teeth \\
Complete & movement) AND (transplantation [MeSH \\
(EBSCO) & Terms] OR tooth autotransplantation OR teeth \\
Cochrane & autotransplantation OR autogenous tooth \\
& transplantation OR autogenous teeth trans- \\
& plantation OR tooth transplantation OR teeth \\
& transplantation) AND (root resorption [MeSH \\
& Terms] OR tooth mobility [MeSH Terms] OR \\
& teeth mobility [MeSH Terms] OR tooth vitality \\
& [MeSH Terms] OR teeth vitality OR survival \\
& rate [MeSH Terms] OR success rate [MeSH \\
& Terms] OR efficacy OR side effects [MeSH \\
& Terms] OR collateral effects)) \\
\hline Trials Central & ((orthodontics [MeSH Terms])) \\
\hline Clinical trials & ((orthodontics [MeSH Terms]) AND \\
& (Orthodontic treatment)) \\
\hline
\end{tabular}

To identify the relevant articles without limitation of year and restriction of language, published up to January 3, 2020, a search was conducted in the following electronic databases: PubMed, Scopus, Web of Science, Medline Complete (EBSCO), Cochrane, Clinical Trials, and Trials Central. Specific journals in the area were also consulted. The descriptors/MeSH terms were used ( - Table $\mathbf{1}$ ).

\section{Eligibility Criteria of the Articles}

The eligibility criteria were determined in accords with the scheme of the patient intervention comparison outcome study (PICOS) question, inclusion, and exclusion criteria (-Table 2).

Initially, the selection of articles was performed by reading the titles and abstracts. Articles in which the title and abstract did not contain sufficient information were fully verified to decide on their eligibility, and repeated articles found in the databases were considered only once.

Two researchers (R.F.C and R.L.S.) performed the selection of articles independently and the results compared with each

Table 2 Criteria (PICOS, inclusion and exclusion) for study selection

\begin{tabular}{|c|c|c|c|c|}
\hline \multicolumn{5}{|c|}{ Patient intervention comparison outcome study } \\
\hline Participants (P) & \multicolumn{4}{|c|}{ Orthodontic patients with complete or incomplete development of tooth roots, with a minimum age of 9 years } \\
\hline Intervention (I) & \multicolumn{4}{|c|}{ Tooth transplantation and orthodontic tooth movement } \\
\hline Comparison (C) & \multicolumn{4}{|c|}{ Effects of orthodontic movement on tooth auto transplantation with a control group } \\
\hline Result $(\mathrm{O})$ & $\begin{array}{l}\text { Bone: presence of lamina } \\
\text { dura, bone resorption, } \\
\text { vestibular thickness, } \\
\text { height and bone promi- } \\
\text { nence, vertical bone loss }\end{array}$ & $\begin{array}{l}\text { Tooth: root resorption, changes } \\
\text { in pulp chamber, tooth vitality, } \\
\text { color change, tooth mobility and } \\
\text { ankylosis, endodontic treatment, } \\
\text { endodontic treatment stability }\end{array}$ & $\begin{array}{l}\text { Periodontium: } \\
\text { pocket depth, } \\
\text { periodontal space, } \\
\text { gingival recession }\end{array}$ & Esthetics: patient satisfaction \\
\hline Study $(S)$ & \multicolumn{4}{|c|}{ RCTs that evaluated transplanted teeth submitted to orthodontic movement } \\
\hline \multicolumn{5}{|l|}{ CRITERIA } \\
\hline Inclusion & \multicolumn{4}{|c|}{$\begin{array}{l}\text { Report survival rate, success, pulp condition, tooth mobility, presence of ankylosis and root resorption of transplanted } \\
\text { teeth with complete or incomplete root formation in orthodontic patients with a mean follow-up period of at least } 1 \text { year }\end{array}$} \\
\hline Exclusion & \multicolumn{4}{|c|}{$\begin{array}{l}\text { Patients with systemic diseases, syndromes, or cleft lip and palate. Studies reporting auto transplantation of teeth } \\
\text { with histories of cysts, tumors, trauma or fistulas. Studies without information about follow-up or root resorption, case } \\
\text { reports and series of cases with fewer than } 15 \text { orthodontic patients, studies with animals, reviews of the literature and } \\
\text { editorials }\end{array}$} \\
\hline
\end{tabular}

Abbreviation: RCT, randomized controlled trail. 
Table 3 Score of each article selected with the inclusion criteria according to the items of MINORS

\begin{tabular}{|c|c|c|c|c|c|c|c|c|c|c|}
\hline $\begin{array}{l}\text { MINORS } \\
\text { score }^{\text {a }}\end{array}$ & $\begin{array}{l}\text { Lagerström } \\
\text { and } \\
\text { Kristerson } \\
(1986)^{7}\end{array}$ & $\begin{array}{l}\text { Andreasen } \\
\text { et a } \\
(1990)^{5}\end{array}$ & $\begin{array}{l}\text { Frenken } \\
\text { et al } \\
(1998)^{11}\end{array}$ & $\begin{array}{l}\text { Bauss } \\
\text { et al } \\
(2003)^{12}\end{array}$ & $\begin{array}{l}\text { Bauss } \\
\text { et al } \\
(2004)^{13}\end{array}$ & $\begin{array}{l}\text { Jonsson } \\
\text { and } \\
\text { Sigurdsson } \\
(2004)^{14}\end{array}$ & $\begin{array}{l}\text { Tanaka } \\
\text { et al } \\
(2008)^{15}\end{array}$ & $\begin{array}{l}\text { Watanabe } \\
\text { et al } \\
(2010)^{6}\end{array}$ & $\begin{array}{l}\text { Kokai } \\
\text { et al } \\
(2015)^{4}\end{array}$ & $\begin{array}{l}\text { Yang et al } \\
(2019)^{16}\end{array}$ \\
\hline $\begin{array}{l}\text { A clearly } \\
\text { stated aim }\end{array}$ & 2 & 2 & 2 & 2 & 2 & 2 & 1 & 2 & 2 & 1 \\
\hline $\begin{array}{l}\text { Inclusion of } \\
\text { consecutive } \\
\text { patients }\end{array}$ & 1 & 2 & 1 & 1 & 2 & 2 & 1 & 1 & 1 & 1 \\
\hline $\begin{array}{l}\text { Prospective } \\
\text { data } \\
\text { collection }\end{array}$ & 2 & 2 & 2 & 2 & 2 & 2 & 2 & 2 & 2 & 2 \\
\hline $\begin{array}{l}\text { End points } \\
\text { appropriate } \\
\text { to the aim of } \\
\text { the study }\end{array}$ & 1 & 2 & 1 & 1 & 1 & 1 & 1 & 1 & 1 & 2 \\
\hline $\begin{array}{l}\text { Unbiased } \\
\text { assessment } \\
\text { of the study } \\
\text { end point } \\
\end{array}$ & 0 & 0 & 0 & 0 & 0 & 0 & 0 & 0 & 0 & 0 \\
\hline \begin{tabular}{|l|} 
Follow-up \\
period appro- \\
priate to the \\
aim of the \\
study \\
\end{tabular} & 1 & 2 & 1 & 1 & 2 & 2 & 2 & 2 & 1 & 1 \\
\hline $\begin{array}{l}\text { Loss of fol- } \\
\text { low-up lower } \\
\text { than } 5 \%\end{array}$ & 2 & 2 & 0 & 0 & 2 & 0 & 1 & 0 & 2 & 2 \\
\hline $\begin{array}{l}\text { Prospective } \\
\text { calculation of } \\
\text { the study size }\end{array}$ & 0 & 0 & 0 & 0 & 0 & 0 & 0 & 0 & 0 & 0 \\
\hline \begin{tabular}{|l|} 
Adequate \\
control group
\end{tabular} & 2 & 1 & 1 & 2 & 2 & 1 & 1 & 1 & 0 & 2 \\
\hline $\begin{array}{l}\text { Contempo- } \\
\text { rary groups }\end{array}$ & 1 & 1 & 0 & 0 & 1 & 0 & 0 & 0 & 0 & 0 \\
\hline $\begin{array}{l}\text { Baseline } \\
\text { equivalence } \\
\text { of groups }\end{array}$ & 1 & 1 & 1 & 1 & 1 & 0 & 0 & 0 & 1 & 1 \\
\hline $\begin{array}{l}\text { Adequate } \\
\text { statistical } \\
\text { analyses }\end{array}$ & 1 & 1 & 1 & 1 & 1 & 1 & 1 & 1 & 1 & 2 \\
\hline Total score & 14 & 16 & 10 & 11 & 16 & 11 & 10 & 10 & 11 & 14 \\
\hline Risk of bias & Medium & Medium & Medium & Medium & Medium & Medium & Medium & Medium & Medium & Medium \\
\hline
\end{tabular}

ane item received score: 0 -when not reported, $1-$ when inadequately reported, and $2-$ when adequately reported. The articles were classified in accordance with their methodological quality into low $(>17)$, medium $(\geq 10 \leq 17)$, and high risk of bias $(<10)$.

other, to avoid divergences that could occur during data collection. In the disagreements found between the two evaluators with respect to the inclusion or exclusion of any study, a third evaluator would be consulted (F.G.C.) to eliminate the discrepancies. The three evaluators were previously calibrated for the analyzes (Kappa: 0.90).

The references cited in the eligible articles were analyzed to verify if there were any studies that had not been previously mentioned by the electronic databases. ${ }^{9}$

\section{Quality and Risk of Bias Assessment}

Data from the selected articles were independently extracted by the two reviewers; for this purpose, they used a personalized version of the Cochrane data extraction model for reviews. ${ }^{10}$
After data collection, the information obtained from each study was organized in tables that examined the PICOS criteria ( - Table 2). Methodological quality of eligible articles was assessed using the MINORS (- Table 3 ) based on the following scores: $0-$ when unreported item, $1-$ when reported inappropriately, and 2-when reported properly. The articles were classified based on their methodological quality: low $(>17)$, medium $(\geq 10 \leq 17)$, and high risk of bias $(<10)$.

\section{Results}

\section{Selection of Studies}

After screening the titles and abstracts of 443 articles, 38 potentially eligible articles were selected for full text 
verification. After analysis, 10 studies were selected to compose this systematic review, as they met the inclusion criteria (-Fig. 1). Lagerström and Kristerson, ${ }^{7}$ Andreasen et al, ${ }^{5}$ Frenken et al, ${ }^{11}$ Bauss et al, ${ }^{12}$ Bauss et al, ${ }^{13}$ Jonsson and Sigurdsson, ${ }^{14}$ Tanaka et al, ${ }^{15}$ Watanabe et $a l,{ }^{6}$ Kokai et $a l,{ }^{4}$ and Yang et al. ${ }^{16}$

All studies were longitudinal, prospective, or retrospective controlled clinical trials that evaluated the results of autotransplantations in orthodontic patients (-Table 3 ).
The articles were evaluated for data extraction in accordance with the PICOS scheme, with a high level of consensus among the reviewers for selecting eligible articles and bias assessments. The results on survival, success, endodontic treatment, ankylosis, and resorption of each study were described (-Table 4), along with items on preoperative assessment, operative protocol, and postoperative assessment of the autotransplantation dental associated with orthodontic movement (-Table 5).

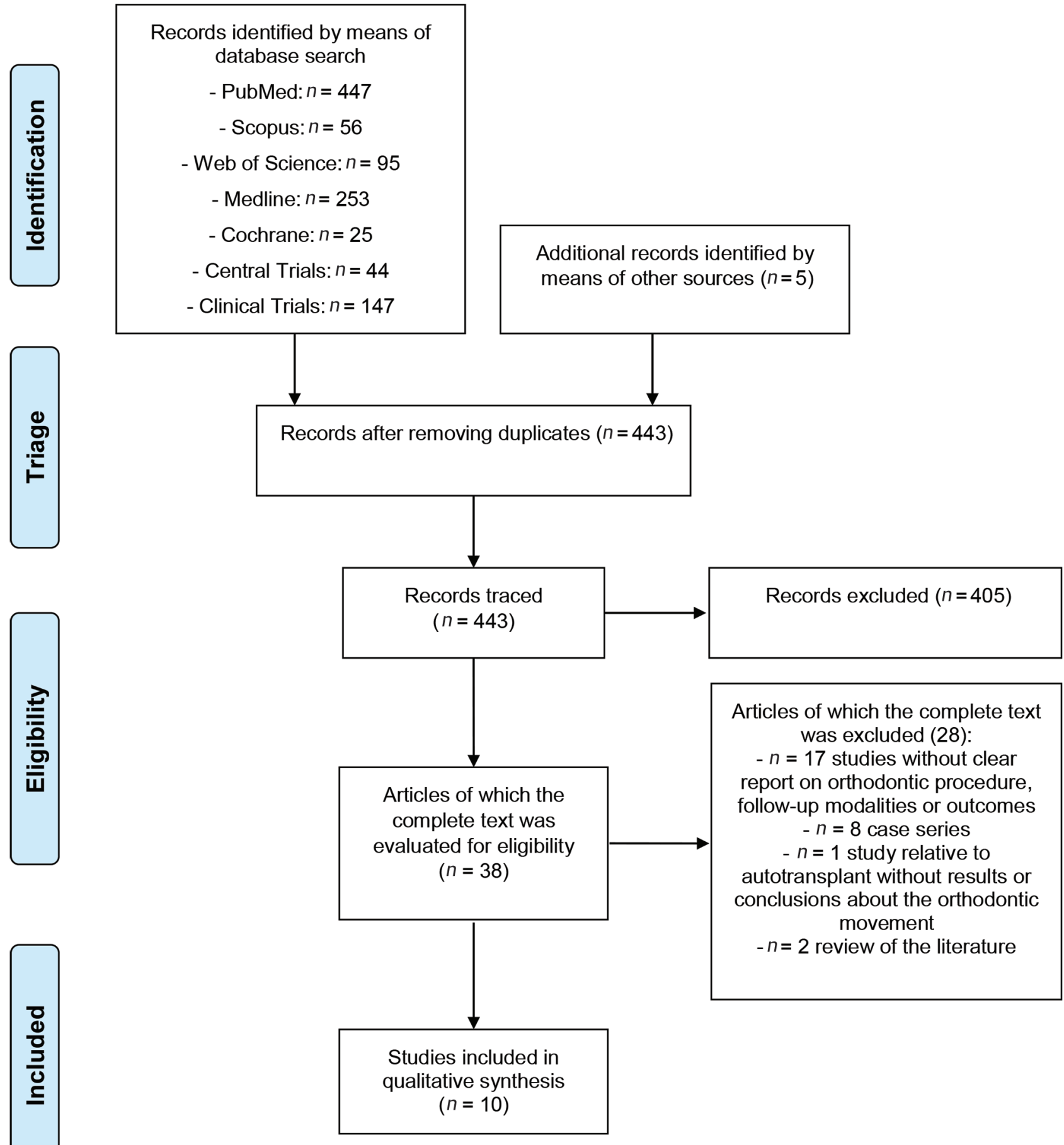

Fig. 1 Flow diagram showing synthesis of the systematic analysis, according to Preferred Reporting Items for Systematic Review and Meta-Analysis (PRISMA) guidelines. 
Effect of Orthodontic Treatment on Tooth Autotransplantation Lacerda-Santos et al. 471

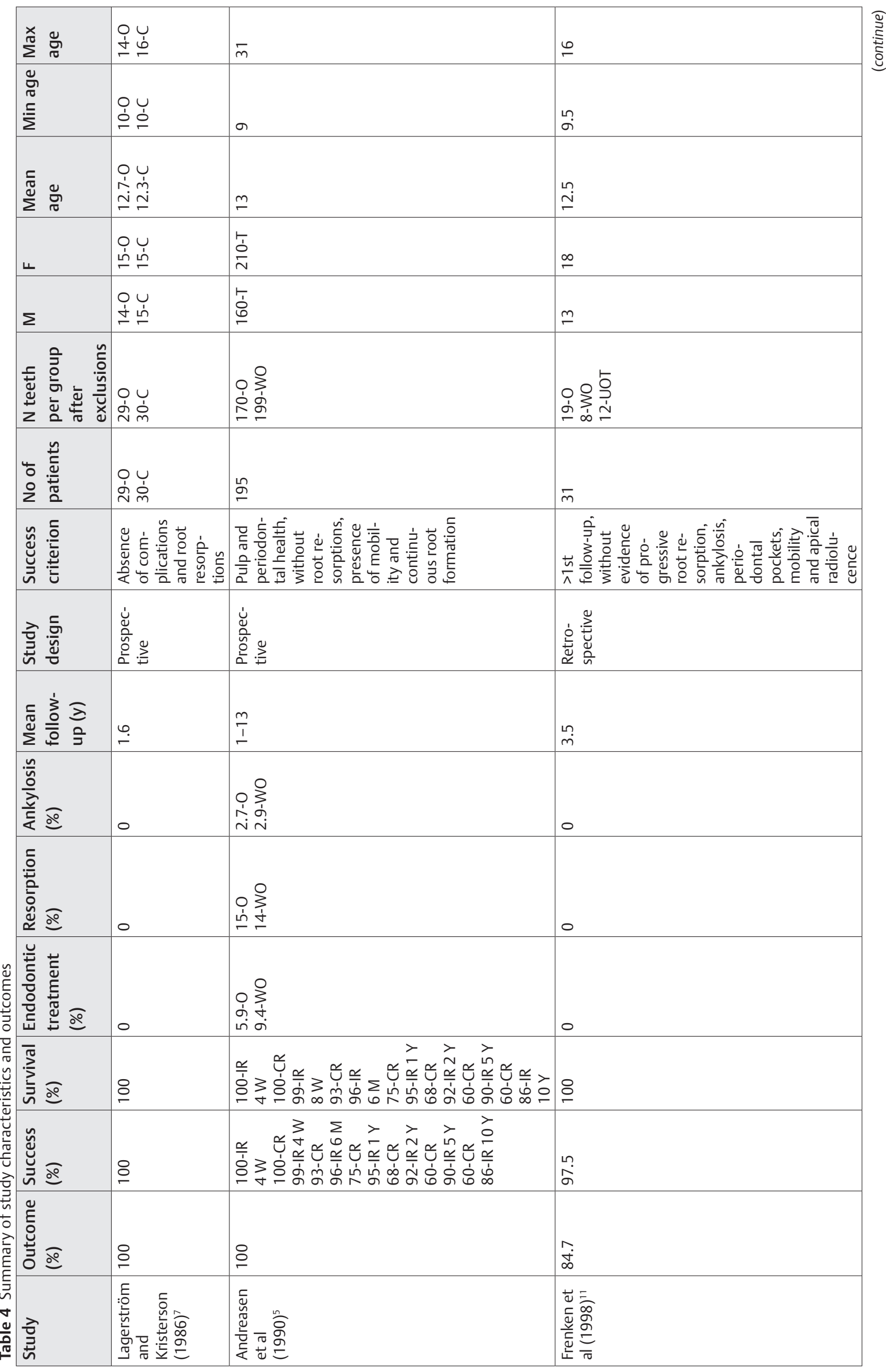




\begin{tabular}{|c|c|c|c|}
\hline$\sum^{x} \underset{\pi}{n}$ & i & $\stackrel{m}{g}$ & gi \\
\hline 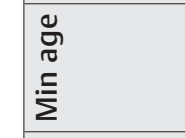 & $\underline{\dot{\Phi}}$ & 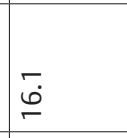 & $\hat{0}$ \\
\hline 离 & $\stackrel{\infty}{=}$ & $\stackrel{m}{\stackrel{2}{2}}$ & $\overline{\dot{m}}$ \\
\hline 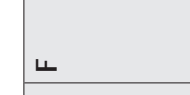 & $\bar{\lambda}$ & $\bar{\sigma}$ & $\cong$ \\
\hline$\Sigma$ & $\circ$ & $i$ & $g$ \\
\hline 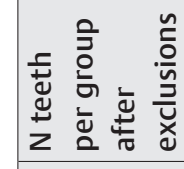 & بِ & 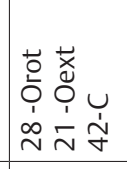 & 品 \\
\hline 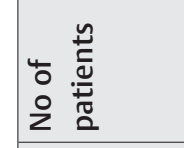 & 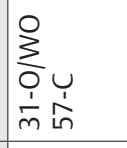 & $\infty$ & $\stackrel{\sim}{\sim}$ \\
\hline 爰 & & & 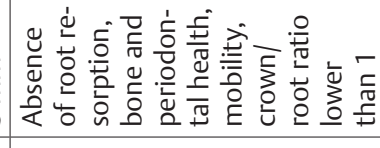 \\
\hline 咅亭 & 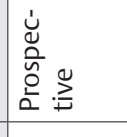 & 冚 & 竞 \\
\hline 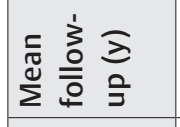 & $\stackrel{\sim}{m}$ & fo & $\stackrel{+}{\stackrel{ \pm}{\circ}}$ \\
\hline$\frac{\frac{n}{0}}{\frac{b}{b}}$ & 号耑 & 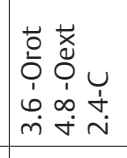 & 8 \\
\hline 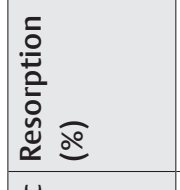 & 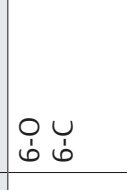 & 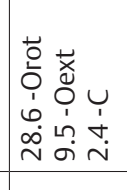 & $\ddot{\circ}$ \\
\hline 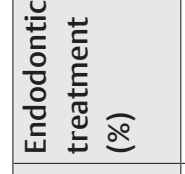 & 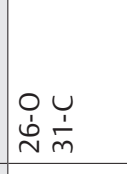 &  & J \\
\hline 㺼 & 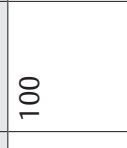 & $\begin{array}{l}0 \\
\dot{\infty} \\
\infty\end{array}$ & 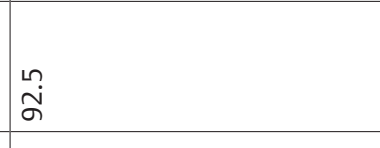 \\
\hline 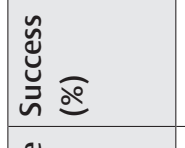 & 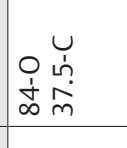 & $\begin{array}{l}0 \\
\dot{\infty} \\
\infty\end{array}$ & 㪿 \\
\hline 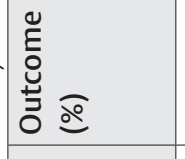 & $\stackrel{8}{\circ}$ & $\ddot{~}$ & 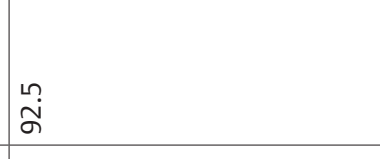 \\
\hline 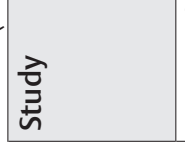 & 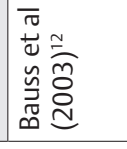 & 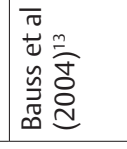 & 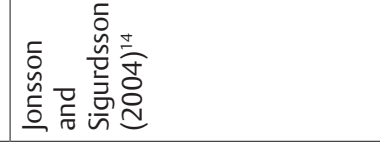 \\
\hline
\end{tabular}


Effect of Orthodontic Treatment on Tooth Autotransplantation Lacerda-Santos et al. 473

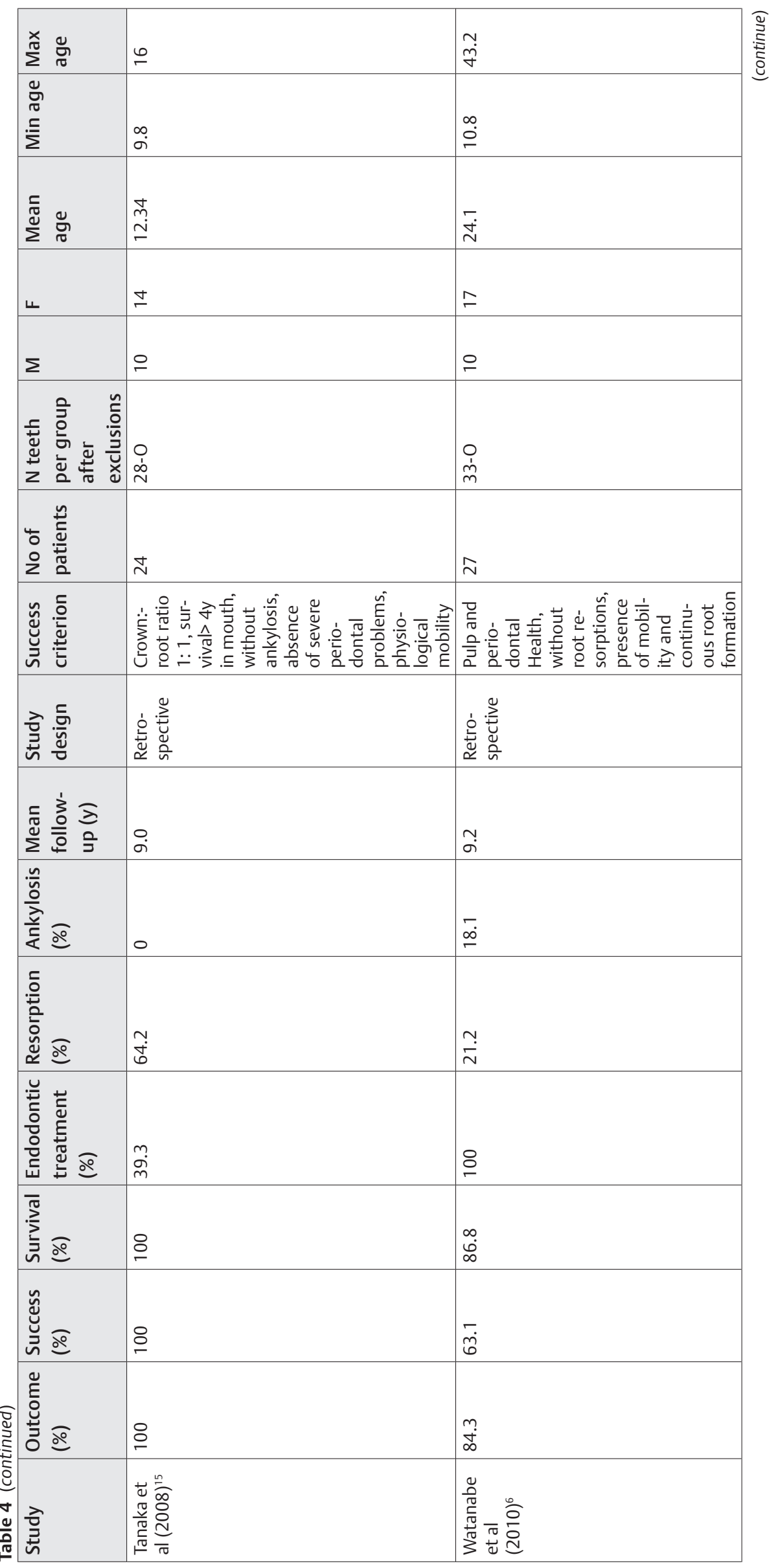




\begin{tabular}{|c|c|c|}
\hline$\sum^{x} \tilde{\pi}$ & $\begin{array}{l}\bar{\infty} \\
\infty \\
n\end{array}$ & in \\
\hline $\begin{array}{l}\tilde{\sigma} \\
\pi \\
.5 \\
\Sigma \Sigma\end{array}$ & $\stackrel{m}{i}$ & $\stackrel{m}{-}$ \\
\hline 离 & হ̄ं & $\stackrel{\sim}{\stackrel{n}{N}}$ \\
\hline\llcorner & 8 & q \\
\hline$\Sigma$ & 인 & ₹ \\
\hline  & $\begin{array}{l}0 \\
0 \\
\dot{1} \\
\end{array}$ & 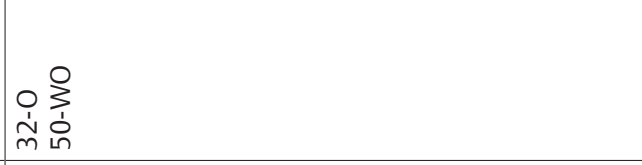 \\
\hline  & $\infty$ & $\approx$ \\
\hline 岕 & 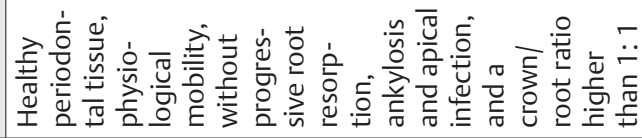 & 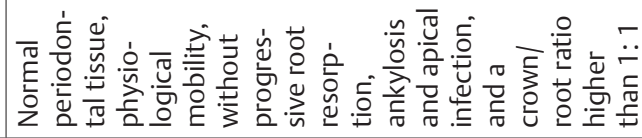 \\
\hline 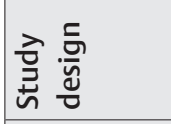 & 事 & 它密 \\
\hline 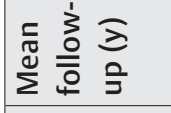 &  & F \\
\hline$\frac{\frac{n}{n}}{\frac{0}{x}}$ & $\simeq$ & 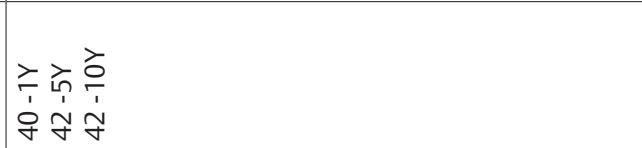 \\
\hline 흘 & $\lambda$ & 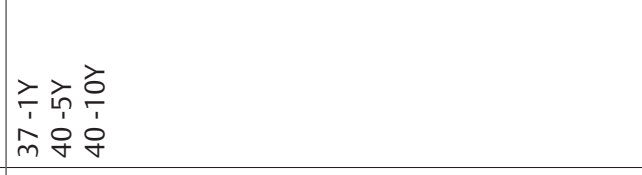 \\
\hline 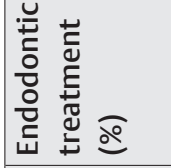 & ঃ & 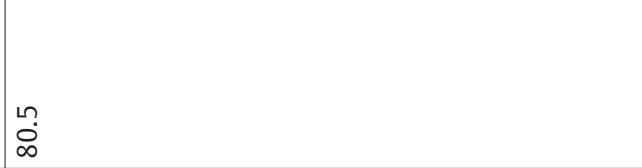 \\
\hline 胥 & n̆ & 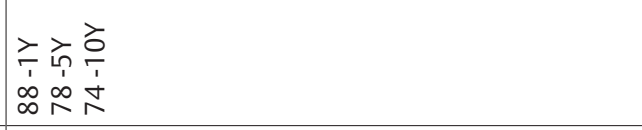 \\
\hline$\stackrel{\breve{u ̈ ~}}{\breve{\Xi}}$ & $\pi$ & 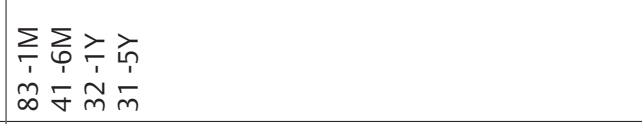 \\
\hline  & 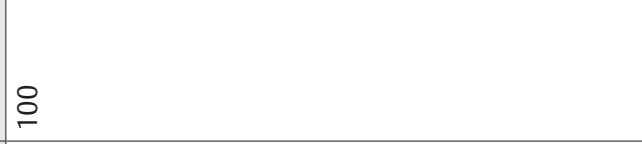 & 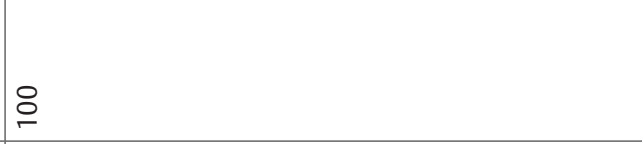 \\
\hline 永 & 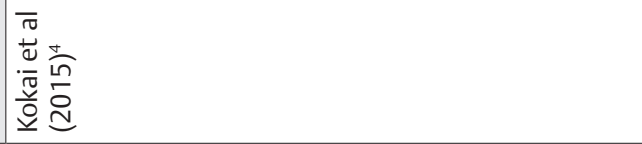 & 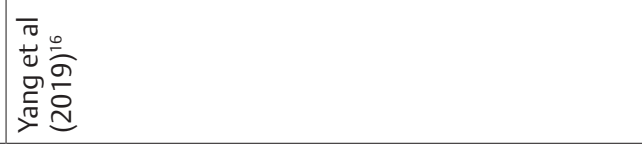 \\
\hline
\end{tabular}


Effect of Orthodontic Treatment on Tooth Autotransplantation Lacerda-Santos et al. 475

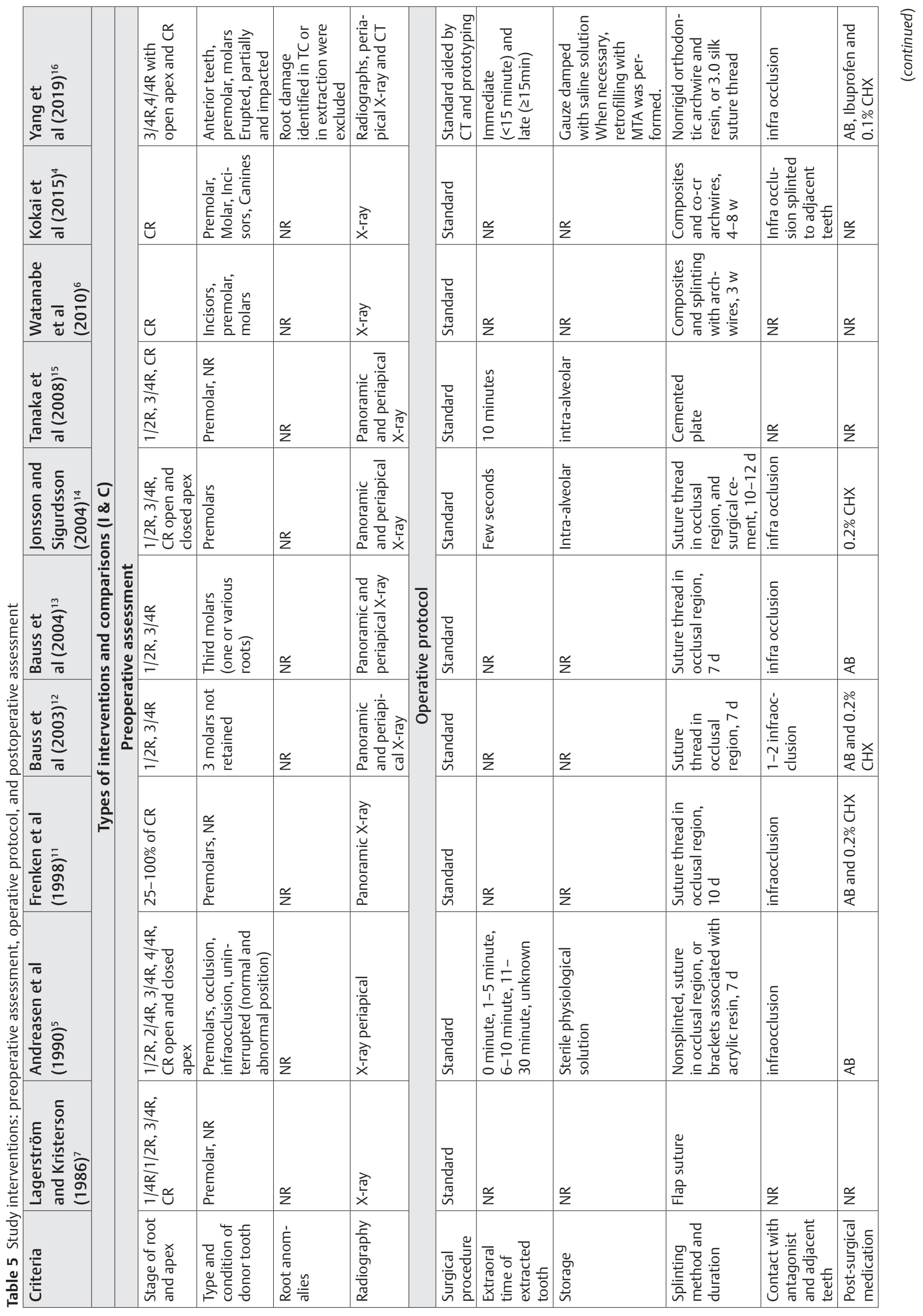




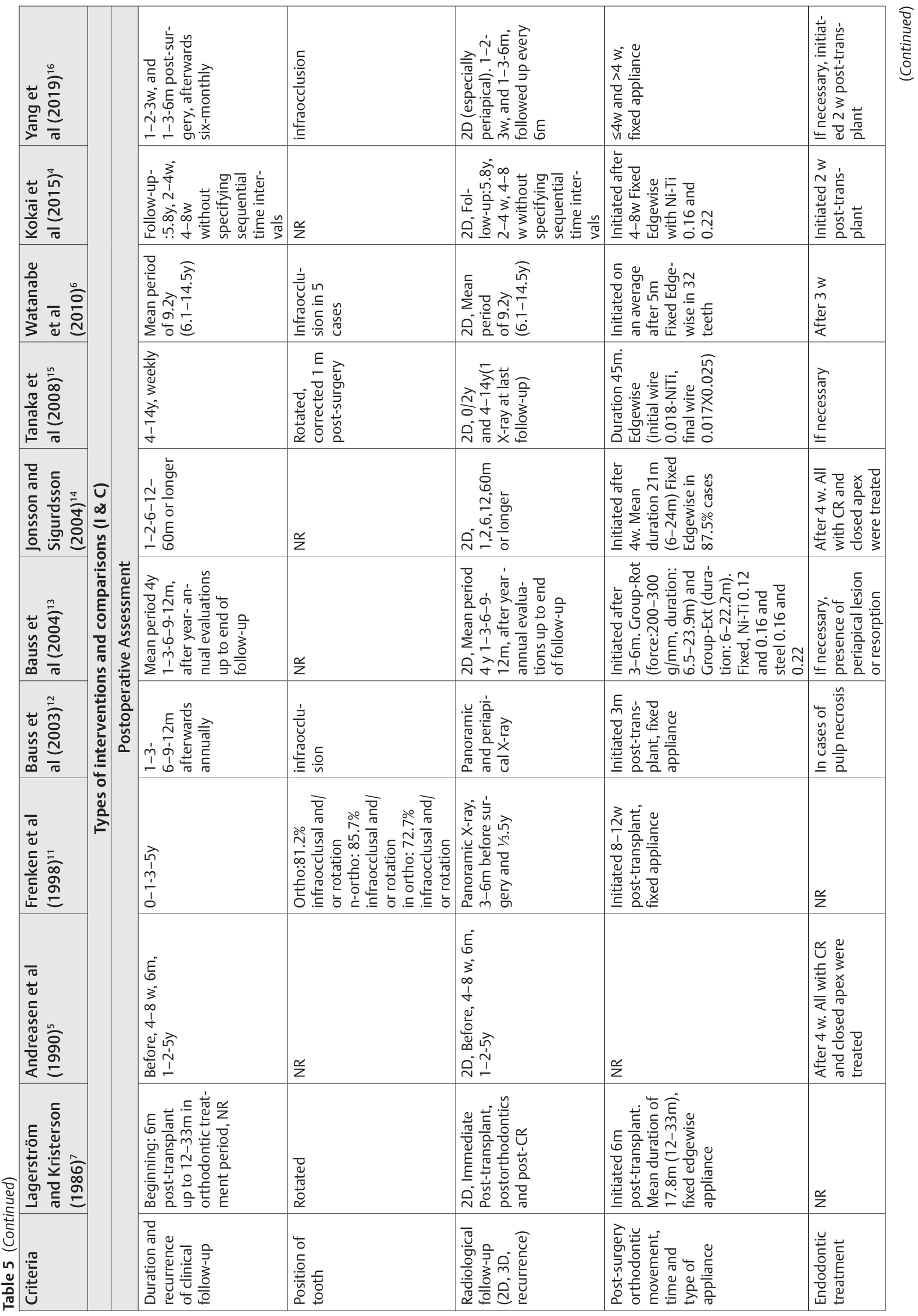


Effect of Orthodontic Treatment on Tooth Autotransplantation Lacerda-Santos et al. 477

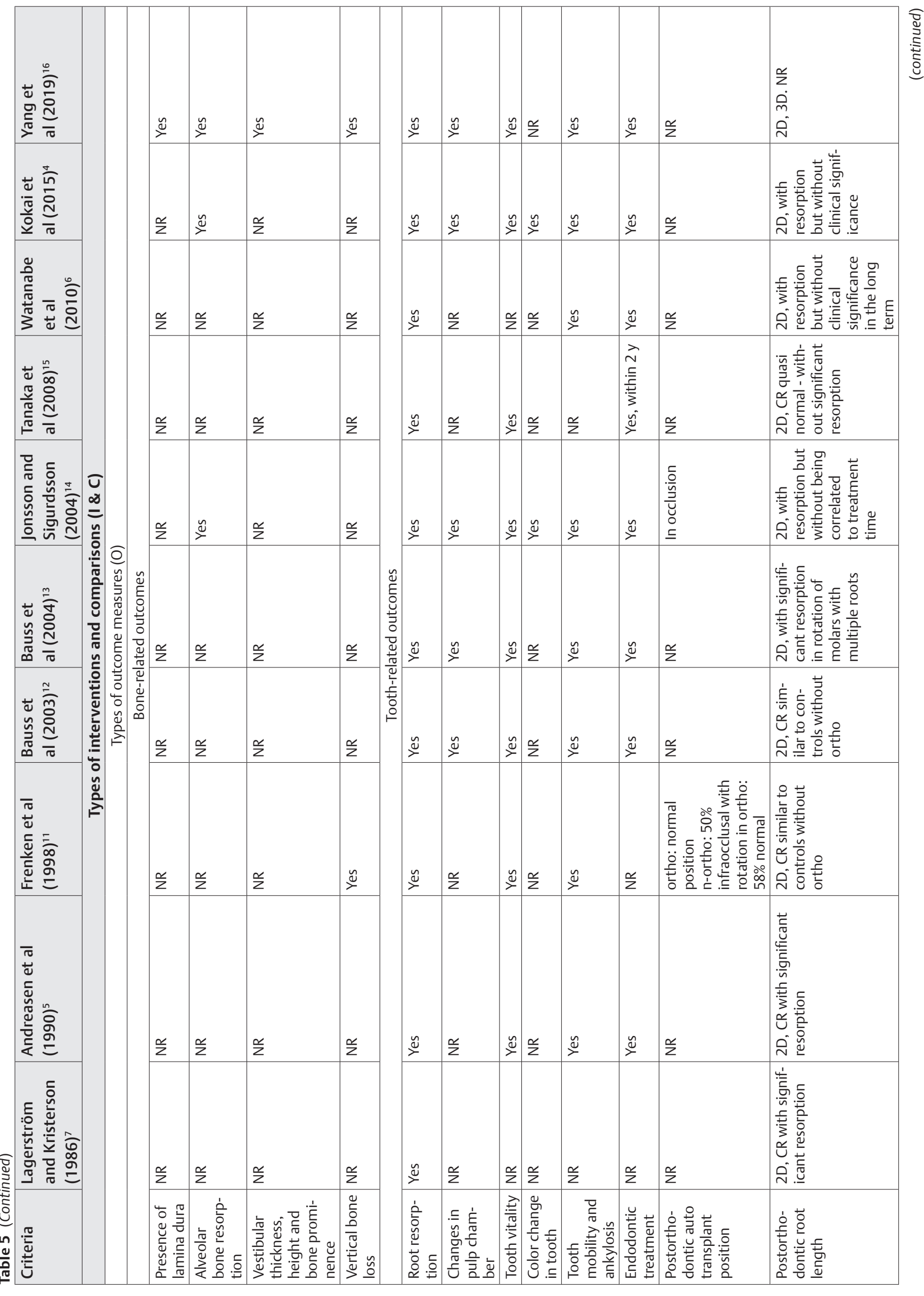




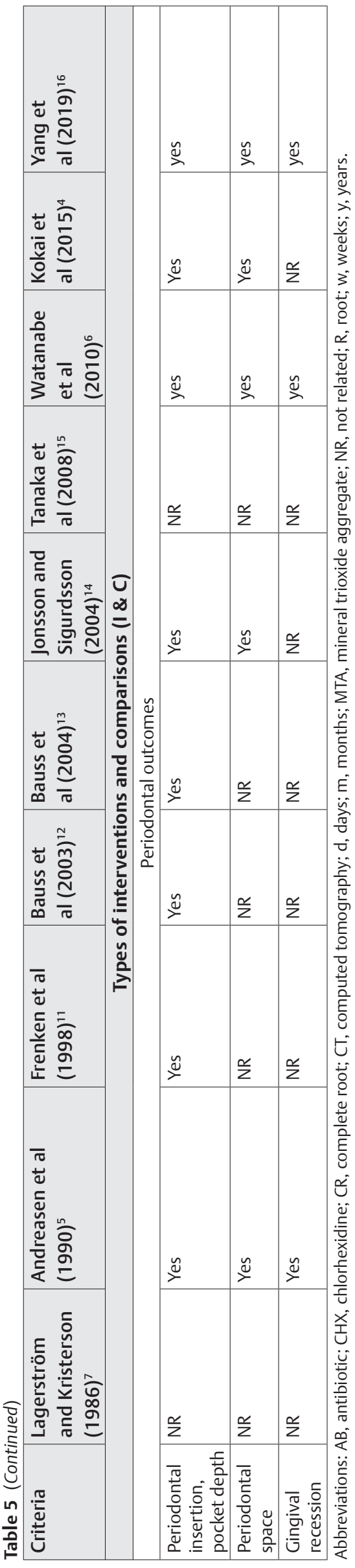

\section{Characteristics of Studies}

All of the studies were written in English, although they had been conducted in different countries such as Sweden, ${ }^{7}$ Denmark, ${ }^{5}$ Holland, ${ }^{11}$ Germany, ${ }^{12,13}$ Iceland, ${ }^{14}$ Japan, ${ }^{4,6,15}$ and South Korea. ${ }^{16}$

Five $^{5,7,12,13,16}$ studies reported the use of control groups with a minimum number of 30 patients with transplanted teeth without orthodontic movement, one study ${ }^{11}$ used only 8 patients as control, and another four studies ${ }^{4,6,14,15}$ used homologous or adjacent nontransplanted teeth as control of the autotransplanted teeth moved. The majority of the studies $^{4-6,11-16}$ used well-defined criteria of success for autotransplants, differently from the study of Lagerström and Kristerson. ${ }^{7}$ None of the studies ${ }^{4-7,11-16}$ mentioned having realized the calculation of sample size and power of the study.

\section{Characteristics of Participants}

The mean age of study participants ranged from $12.3^{7,15}$ to 29.1 years $^{4}$ (minimum age 9 years $^{5}$ and maximum 58.1 years ${ }^{4}$ ). Gender distribution was presented in the studies. ${ }^{4-7,11-16}$ Between $24^{15}$ and 195 patients ${ }^{5}$ participated in each study. All articles reported the number of transplanted teeth.

In total 579 premolars were used in the studies ${ }^{4-7,11,14-16}$; 346 were transplanted in the groups with orthodontic treatment, ${ }^{4-7,11,14,15} 207$ in the groups without undergoing orthodontic treatment, ${ }^{5,11} 12$ in the group undergoing orthodontic treatment, ${ }^{11}$ and another 14 did not define the group. ${ }^{16}$ In total 282 molars were used $4,6,12,13,16 ; 115$ in groups undergoing orthodontic treatment, $6,12,13,15119$ in groups without orthodontic treatment, ${ }^{12,13}$ and another 48 had no definition of the group. ${ }^{16}$ A total of 43 anterior teeth were also used ${ }^{4,6,16} ; 23$ in groups with orthodontic treatment $\mathrm{t}^{4,6,16}$ and another 20 teeth had no definition of the group. ${ }^{16}$

\section{Characteristics of Interventions \\ Preoperative Assessment}

The studies ${ }^{4-7,11-16}$ did not report the preclinical evaluation in detail, but all of them mentioned the use of radiography. The stage of root formation was described following the criteria of Moorrees et al, ${ }^{17}$ by all the studies. ${ }^{4-7,11-16}$ The autotransplanted teeth had different stages of root and apex formation in all the studies evaluated. ${ }^{4-7,11-16}$ Only two ${ }^{12,13}$ studies did not have autotransplanted teeth with complete roots in the groups at the time of surgery ( - Table $\mathbf{5}$ ).

The condition of the donor tooth was reported in only three ${ }^{5,12,16}$ studies. Angulation of the tooth and the existence of previous orthodontic treatment were not reported in any of the studies. Root anomalies were not mentioned by nine ${ }^{4-7,11-15}$ studies; one ${ }^{16}$ study only reported the exclusion of damaged teeth identified before or during the surgical procedure.

In all the studies, two-dimensional (2D) was mentioned; five ${ }^{12-16}$ used panoramic and periapical X-ray; one ${ }^{11}$ only panoramic X-ray and another, only the periapical type. ${ }^{5}$ Another three studies ${ }^{4,6,7}$ did not mention the radiography type and one ${ }^{16}$ of the studies used both 2D and three-dimensional (3D) (cone-beam computed tomography [CBCT]) analyses. 
The studies reported the indications for dental autotransplantation; seven ${ }^{4,6,7,11-14}$ vaguely described the interrelationship between autotransplantation and orthodontics.

One study ${ }^{15}$ questioned the standard indication of the premolar for orthodontic patients, another two ${ }^{14,16}$ studies described the positive impact of the use of autotransplanted teeth in orthodontic patients without mention the best donor tooth. Another study ${ }^{5}$ only cited the orthodontic movement.

\section{Operative Protocol}

The studies ${ }^{4-7,11-16}$ followed a pattern similar to the surgical technique standardized by Andreasen et al. ${ }^{5}$ Four ${ }^{5,14-16}$ studies reported the extraoral time of the extracted tooth, which was a few seconds, ${ }^{5,14} 1$ to 5 minutes, ${ }^{5} 6$ to 10 minutes, ${ }^{5,15} 11$ to 30 minutes, ${ }^{5}<15$ minutes (immediate), ${ }^{16}$ and $\geq 15$ minutes (late). ${ }^{16}$ Another three ${ }^{7,11,12}$ studies did not report the extraoral time and in one ${ }^{5}$ part of the sample was unknown (-Table 5). One study ${ }^{16}$ mentioned having performed retrofilling with mineral trioxide aggregate at this stage in cases with the need for increasing the extraoral time to $\geq 15 \mathrm{~min}$.

Four ${ }^{5,14-16}$ studies reported the means of storage of the tooth; one ${ }^{16}$ by sing gauze dampened with saline solution, another ${ }^{5}$ physiological solution, and two $\mathrm{O}^{14,15}$ stored the tooth intra-alveolarly in the site of the donor. Another six ${ }^{4,6,7,11-13}$ studies did not report about the existence of and/or storage medium.

All ${ }^{4-7,11-16}$ the studies reported the splinting method; six ${ }^{5,11-}$ ${ }^{14,16}$ used suture thread in the occlusal area of the transplant, two $0^{4,6}$ used composites associated with archwires, one ${ }^{7}$ used the flap suture for stabilization, another ${ }^{15}$ used a cemented occlusal plate, and one ${ }^{5}$ did not splint part of the sample, or used brackets associated with acrylic resin. ${ }^{5}$

The majority of the studies ${ }^{4-6,11-14}$ mentioned the time of using splinting, 7 days, ${ }^{5,12,13} 10$ to 12 days, ${ }^{11,14} 3$ weeks, ${ }^{6}$ and 4 to 8 weeks. ${ }^{4}$ Seven ${ }^{4,5,11-14,16}$ studies reported that occlusal positioning of the transplanted tooth during the surgical procedure was in infraocclusion, from 1 to $2 \mathrm{~mm}^{12}$ short of the occlusal plane; three $e^{6,7,15}$ did not discuss occlusal positioning and none of the studies mentioned the use of the orthodontic appliance before surgery.

\section{Postoperative Assessment}

The majority of studies detailed postoperative and radiographic assessment and none of the studies discussed the postoperative cure protocol for the patients with dental autotransplant.

The duration and recurrence of follow-up were reported in all the studies (-Table 5). The mean duration of follow-up was from 1.6 years, ${ }^{7} 3$ to 4 years, ${ }^{11-13,16} 5.8$ years, ${ }^{4}$ and over 9 yea rs. ${ }^{5,6,14,15}$

All studies used 2D exams for radiographic follow-up; of which, eight t-6,11,13-16 had a duration similar to that of the clinical follow-up. One ${ }^{7}$ study mentioned a distinct clinical radiographic follow-up time, and two $^{4,12}$ did not report the time when the 2D exams were performed.

Relative to the position of the transplanted teeth, three $\mathrm{e}^{6,12,16}$ studies mentioned that they were in infraocclusion, in another two ${ }^{7,15}$ they were rotated, and ${ }^{11}$ one study mentioned that there were teeth in infraocclusion and/or rotated.

Endodontic treatment was performed only in the case of pulp necrosis in one ${ }^{12}$ study; another five $e^{4,6,13,15,16}$ explained that endodontic treatment was performed in the presence of signs of periapical infection or inflammatory root resorption. Two studies ${ }^{5,14}$ reported endodontic treatment in all the teeth with complete roots, and one ${ }^{7}$ study did not report any endodontic treatment.

All the studies performed orthodontic treatment in the postoperative period in at least one group of patients; seven ${ }^{4,6,7,11-14}$ distinctly mentioned the adequate time for beginning with treatment, such as 4 weeks $^{14}$ or 1 to 2 months ${ }^{4}$ or 2 to 3 months $^{11}$ or 3 months $^{12}$ or 3 to 6 months $^{13}$ or 5 to 6 months ${ }^{6,7}$ post-transplantation.

The mean duration of orthodontic treatments was mentioned as being $\leq 4$ and $>4$ weeks, ${ }^{16} 17.8$ months, ${ }^{7} 21$ months, ${ }^{14}$ between 6 and 23 months, ${ }^{13}$ and 45 months. ${ }^{15}$ Another five ${ }^{4-}$ $6,11,12$ studies did not mention the time of duration of treatment. All of the studies used a fixed orthodontic appliance; five $e^{4,6,7,14,15}$ of them mentioned the used of the Edgewise type of mechanics.

\section{Characteristics of the Measurements of Results}

Nine ${ }^{4-6,11-16}$ studies mentioned a set of criteria for success and survival of the transplant, which showed similarities among them, such as absence of periodontal problems, physiological mobility, without progressive root resorption, ankylosis, and apical infection. In addition, ${ }^{12}$ one study cited the existence of occlusal contacts as being a criterion, and four ${ }^{4,14-16}$ cited the proportion of the crown:root ratio, two ${ }^{11,12}$ cited the need for $>1$-year follow-up, and another ${ }^{15>4 \text { year }}$ s survival in the mouth. One ${ }^{7}$ study did not clearly mention the criteria it used for measuring the success of the transplant. For seven ${ }^{5,7,11-15}$ studies noncompliance with one of these criteria lead to the case being considered a failure. Three ${ }^{4,6,16}$ studies considered a factor of success to be that the teeth were still present and functioning well at the end of follow-up period, and that this should also be included in the survival rate.

The result most broad in scope related to bone was mentioned by only one ${ }^{16}$ study; two ${ }^{4,14}$ reported alveolar bone resorption, and another ${ }^{11}$ reported only about vertical bone loss (-Table 5 ).

The results with reference to the teeth were reported more frequently (-Table 5). All of the studies discussed the rate of root resorption, the majority ${ }^{4,5,11-16}$ reported vitality tests in the

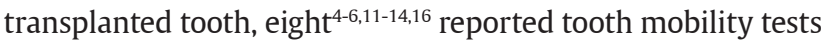
and later ankylosis, and eight ${ }^{4-6,12-16}$ reported on the frequency (-Table 4) and performance of endodontic treatment.

Only five $e^{4,12-14,16}$ studies mentioned changes in the pulp chamber appearance, and two ${ }^{4,14}$ discussed color changes or compared the color of the teeth.

Only two ${ }^{11,14}$ studies discussed the stability and frequency of the transplanted tooth postorthodontic treatment (-Table 5). All of the studies evaluated the root length of the transplant postorthodontic treatment, nine $e^{4-7,11-15}$ with 2D exams and one used ${ }^{16} 2 \mathrm{D}$ and 3D. Only one ${ }^{16}$ study did not 
discuss the relevance of root resorption of autotransplanted teeth that were orthodontically moved.

In the soft tissue evaluations, eight ${ }^{4-6,11-14,16}$ studies clinically verified the periodontal fixation with reference to pocket depth; five ${ }^{4-6,14,16}$ studies evaluated the periodontal space, and three studies ${ }^{5,6,16}$ evaluated the gingival recession levels (-Table 5).

None of the studies evaluated reported tests to the evaluation of the satisfaction of patients who received the transplants, or the association of autotransplants and orthodontic treatment. No objective criterion was used in the studies to score the esthetic result, either by the patient, surgeon, or the orthodontist responsible for transplantation of the tooth.

\section{Mean Duration of Follow-Up}

Follow-up was reported by all of the studies-7,11-16; only one ${ }^{7}$ had a mean period ( 1.6 years $)^{7}$ shorter than 3 years; the oth$\mathrm{er}^{4-6,11-16}$ studies reported periods of 3.2 to 10.4 years. When considering existing subgroups, only those with a minimum mean follow-up time of 12 months were considered for analysis.

\section{Quality Evaluation}

All of the studies were considered of mean methodological quality in accordance with the MINORS criteria, obtaining scores of 10 points, ${ }^{6,11,15} 11$ points,, ${ }^{4,12,14} 14$ points, ${ }^{7,16}$ and 16 points $^{5,13}$ (-Table 3 ).

The main items not filled out for the MINORS criteria, which showed bias, were the impartial evaluation of the study outcomes, ${ }^{4-7,11-16}$ sample loss to follow-up lower than $5 \%,{ }^{6,11,12,14}$ prospective sample size calculation necessary for the study, ${ }^{4-7,11-16}$ presence of contemporary groups evaluated, ${ }^{4,6,11,12,14-16}$ and equivalence of initial characteristics among the groups. ${ }^{6,14,15}$

\section{Discussion}

\section{Summary of Evidence}

Significant absence of bias of diagnosis was described in postoperative planning, and deficiencies in the operative and postoperative protocol were observed. Although there was greater uniformity among the studies with respect to the surgical technique, none of the studies clearly reported the angulation/intraosseous position and careful manipulation of the donor tooth, as well as previous orthodontic treatment, which could have had impact on the quality and quantity of success of the autotransplanted teeth.

Qualitative-quantitative outcomes of the alveolar bone and periodontal condition, position of the transplant postorthodontic treatment, and feedback from the patients relative to their satisfaction with the result and experience of treatment were not discussed by the studies to support any form of treatment decision.

There was an effort in the sense of including high-quality studies; however, the majority of studies found were retrospective ${ }^{4,6,11,14-16}$ (- Table 4), and all of them ${ }^{4-7,11-16}$ with a high level of bias, indicating the need for better designed projects to obtain clinical answers.
A high degree of heterogeneity in the methodological design and interventions occurred among the studies-7,11-16; differences in relation to the number of teeth, age, patients, and controls with their initial situations, distinct interventions, unclear approach to appliance, and distinctly asymmetrical treatment times during the outcome made it impossible to analyze the data qualitatively in the form of a metanalysis.

None of the studies included reported adequate guidelines for clinical and radiographic evaluation of the bony bed, or perimeter of the arch and dental positions, or discussion of orthodontic pretreatment.

The studies s-711-16 $^{4}$ described the use of $2 \mathrm{D}$ exams to analyze the stage of root development and dental apex, but did not mention the angulation nor specify the root anomalies. Exams in 3D with CBCT for diagnosis and structured planning of the teeth and bone were cited by only one ${ }^{16}$ study in 2019. However, it is necessary to emphasize that the majority $^{5,7,11-14}$ of the studies evaluated were conducted before 2004, at a time when 3D exams were not routinely performed in the clinic. Nevertheless, it is necessary to recognize that 3D exams could have an important status in the final clinical treatment decision, and must be encouraged as a means of diagnosis ${ }^{18}$ for greater surgical precision, ${ }^{16}$ bone evaluation, and orthodontic decision making.

All studies ${ }^{4-7,11-16}$ addressed the same type of surgical technique, when this was reported, and sought to minimize the extraoral time of the autotransplant. ${ }^{5,14-16}$ The authors basically reported the use of saline solution, ${ }^{16}$ physiological solution, ${ }^{5}$ or intra-alveolar storage. ${ }^{14,15}$

The greatest variations occurred in the splinting method (flap suture, ${ }^{7}$ suture thread,, ${ }^{5,11-14,16}$ archwires and resin,,6,16 cemented acrylic plate, ${ }^{15}$ brackets and acrylic resin ${ }^{5}$ ) and different times and duration, such as 7 days $^{5,12,13}$ or 10 to 12 days $^{11,14}$ or 3 weeks ${ }^{6}$ or 4 to 8 weeks, ${ }^{4}$ without clearly discussing the best method and time.

In the postoperative period, clinical evaluation was followed up during orthodontic treatment in all ${ }^{4-711-16}$ studies; however, the duration reported varied from a short period of time with a mean of a few weeks to months, ${ }^{16}$ intermediate period such as 1 to 2 years, ${ }^{7,13,14}$ longs periods of over 3 years ${ }^{15}$ through to studies that did not cite the duration of fixed orthodontic treatment. ${ }^{4-6,11,12}$ An important, but clinically not significant relationship, was found between pulp necrosis and orthodontic treatment duration. ${ }^{13}$

Exclusively 2D radiographic follow-up was performed among the studies and for similar times to those of the clinic, in the majority, ${ }^{5,6,11,13-16}$ differing from the clinical type in one ${ }^{7}$ and not clearly reported in two studies. ${ }^{4,12}$ Some studies ${ }^{12,13,15}$ considered endodontic treatment only if necessary during follow-up; others ${ }^{5,14}$ performed it in all the teeth with complete roots and/or closed apices. One ${ }^{16}$ study reported earlier treatment, although the authors tended to wait for a good potential pulp response, ${ }^{12,15,16}$ and in other ${ }^{4,6,7,11}$ studies the endodontic treatment strategy was not clear.

According to the majority of the studies, ${ }^{5,7,11-16}$ the teeth with open apices ${ }^{4,5,12,15,16}$ allow better perspectives for longterm success when compared with those with closed apices $^{5,14,16}$ therefore, the large majority of the autotransplanted 
teeth were in this condition. Teeth with multiple roots were associated with increase in pulp with compromised vascular and nerve conditions. ${ }^{13}$ However, authors ${ }^{19}$ have shown the need to reevaluate the endodontic protocol for uniradicular autotransplants with already closed apices, considering its potential for revascularization.

All of ${ }^{4-7,11-16}$ the studies reported more characteristics with reference to the teeth, such as survival, success, endodontic treatment, resorption, ankylosis, and orthodontic treatment. Changes in the pulp changes, changes in tooth color, and position of the autotransplanted tooth in the postorthodontic period were the items less frequently approached. Only two ${ }^{4,14}$ studies reported a more ample clinical evaluation, and three $e^{5,6,16}$ studies made a more extensive evaluation of the periodontal results. None of the studies evaluated discussed the esthetic requirements found by the patients.

Although there is a consensus that orthodontic treatment is important for adequate treatment of the teeth, ${ }^{4,711-16}$ in the dental arch, complications such as root resorption may increase in the transplanted tooth. ${ }^{5-7,13,16}$ Therefore, further studies must make an effort to describe the parameters of standard clinical results to obtain greater success. The criteria used for evaluating the final result in autotransplantation associated with orthodontic treatment were most variable, ranging from intraosseous presence of the tooth through to its postorthodontic treatment position with adequate function, without resorption, ankylosis and with vitality. ${ }^{4,6}$ The eventual loss of the transplanted tooth postorthodontic treatment occurring due to the movement or associated with it must be considered. Although it is not something desired, it will occur due to resorption or bone substitution, ${ }^{20}$ preserving the dimensions of the bony bed, which are improvements in its presurgical condition, making it possible to insert dental implants or new perform orthodontic movement to the site, which was not possible before. In this context, failure of the transplant may result in most successful increase in alveolar bone volume; therefore, both the survival (presence of the tooth) and success (satisfaction with the criteria that define success) must always be considered. ${ }^{4,6,16}$

Three ${ }^{6,12,16}$ studies reported higher rates of ankylosed teeth, with the occurrence of $18.1 \%^{6}$ to $42 \%{ }^{16}$ in a period of 10 years.

Higher rates of ankylosis have been verified when traumatic lesions to the periodontal ligament occur ${ }^{4,5,16}$, in the surgical procedure, or due to endodontic treatment $t^{4,6}$ performed extra-alveolarly ${ }^{16}$, which stimulates bone formation directly on the dentin, root resorption followed by progressive bone substitution until complete resorption occurs with a survival close to 20 years, ${ }^{21}$ with more intense effect in youngsters and slower in adults. ${ }^{21}$

Root resorption was reported in seven ${ }^{4-6,12,13,15,16}$ studies, with occurrence of $6^{12}$ to $64 \%{ }^{15}$ additional rates have been associated with orthodontic treatment. ${ }^{5,7,13,15}$ Authors have reported that the excess of early orthodontic force, ${ }^{4}$ correction of rotation and teeth with multiple roots ${ }^{13}$ could significantly increase resorption. ${ }^{4,13}$ However, the studies did not exhaustively discuss the quantity of this resorption; in the set, the majority ${ }^{4-7,11-14}$ of the studies were unanimous about the low significance and clinical relevance of the increase in root resorption arising from orthodontic movement, justified by the lower rates of ankylosis, ${ }^{4-711-15}$ better position of the tooth, and occlusal function in the $\operatorname{arch}^{4}$ when compared with autotransplanted teeth that were not moved. ${ }^{12,13}$ But in the presence of root lesion/infection of the transplant during orthodontic movement, the studies did not discuss the suspension of movement, wait for regression of postendodontic treatment infection, and the time for finalizing orthodontic treatment.

\section{Implications for Practice}

Greater standardization is necessary for the clinical evaluation parameters, clinical advantages, and risks of orthodontic movement of autotransplanted teeth, and an approach to esthetic satisfaction and quality of life of patients submitted to this association of treatment.

Sufficient clinical evidence has been shown to justify dental autotransplantation at different stages of root development in patients undergoing orthodontic treatment. Root resorption of autotransplanted teeth increased, but it was not considered a limiting factor for treatment; and ankylosis diminished in the orthodontic patients.

\section{Implications for Research}

Randomized controlled trials (RCTs) with sample calculations to support more robust conclusion are recommendable. However, due to the particularities of autotransplantation, in addition to factors such as age, patient expectations, prosthetic rehabilitation options, and orthodontic planning, may make it difficult to conduct RCTs. Therefore, future high-quality longitudinal observational studies may allow significant outcomes to be obtained.

More substantial approaches are needed to the relationships between the type of tooth, its localization, and best indication for orthodontic patients. Planning in 3D and detailed parameters relative to survival versus clinical success in the long-term need to be approached.

\section{Conclusions}

- The quality of the set of evidences found was considered medium due to the existence of methodological problems, risk of bias, and heterogeneity in the eligible articles. There was a sufficient body of evidence that justified autotransplantation in patients who needed orthodontic movement.

- In teeth, there was an increase in root resorption influenced by orthodontics, but without impacting on the general clinical result in the long term.

- Bone and periodontal tissue do not appear to be significantly affected by orthodontics, but they have not been sufficiently addressed.

- The patient's aesthetic satisfaction was not considered in the studies. 


\section{Conflict of Interest}

None declared.

\section{References}

1 Almpani K, Papageorgiou SN, Papadopoulos MA. Autotransplantation of teeth in humans: a systematic review and meta-analysis. Clin Oral Investig 2015;19(6):1157-1179

2 Schatz JP, Joho JP. Indications of autotransplantation of teeth in orthodontic problem cases. Am J Orthod Dentofacial Orthop 1994;106(4):351-357

3 Terheyden $\mathrm{H}$, Wüsthoff $\mathrm{F}$. Occlusal rehabilitation in patients with congenitally missing teeth-dental implants, conventional prosthetics, tooth autotransplants, and preservation of deciduous teeth-a systematic review. Int J Implant Dent 2015;1(1):30

4 Kokai S, Kanno Z, Koike S, et al. Retrospective study of 100 autotransplanted teeth with complete root formation and subsequent orthodontic treatment. Am J Orthod Dentofacial Orthop 2015;148(6):982-989

5 Andreasen JO, Paulsen HU, Yu Z, Schwartz O. A long-term study of 370 autotransplanted premolars. Part III. Periodontal healing subsequent to transplantation. Eur J Orthod 1990;12(1):25-37

6 Watanabe Y, Mohri T, Takeyama M, et al. Long-term observation of autotransplanted teeth with complete root formation in orthodontic patients. Am J Orthod Dentofacial Orthop 2010;138(6):720-726

7 Lagerström L, Kristerson L. Influence of orthodontic treatment on root development of autotransplanted premolars. Am J Orthod 1986;89(2):146-150

8 Liberati A, Altman DG, Tetzlaff J, et al. The PRISMA statement for reporting systematic reviews and meta-analyses of studies that evaluate health care interventions: explanation and elaboration. J Clin Epidemiol 2009;62(10):e1-e34

9 Lacerda-Santos R, Bravin TC, Carvalho FG. Pithon MM, Lima ABL, da Silva KG. Efficacy of topical anesthetics in pain perception during mini-implant insertion: systematic review of controlled clinical trials. Anesth Prog 2019;66(3):119-132

10 Higgins JPT, Green S. Cochrane Handbook for Systematic Reviews of Interventions [updated March 2011] [Version. 5.1.0]. The Cochrane Collaboration, Table 7.7 a: formulae for combining groups. Available at: www.training.cochrane.org/ handbook. Accessed April 3, 2020

11 Frenken JW, Baart JA, Jovanovic A. Autotransplantation of premolars. A retrospective study. Int J Oral Maxillofac Surg 1998;27(3):181-185

12 Bauss O, Sadat-Khonsari R, Engelke W, Kahl-Nieke B. Results of transplanting developing third molars as part of orthodontics space management. Part 2: results following the orthodontic treatment of transplanted developing third molars in cases of aplasia and premature loss of teeth with atrophy of the alveolar process. J Orofac Orthop 2003;64(1):40-47

13 Bauss O, Schwestka-Polly R, Kiliaridis S. Influence of orthodontic derotation and extrusion on pulpal and periodontal condition of autotransplanted immature third molars. Am J Orthod Dentofacial Orthop 2004;125(4):488-496

14 Jonsson T, Sigurdsson TJ. Autotransplantation of premolars to premolar sites. A long-term follow-up study of 40 consecutive patients. Am J Orthod Dentofacial Orthop 2004;125(6):668-675

15 Tanaka T, Deguchi T, Kageyama T, Kanomi R, Inoue M, Foong KW. Autotransplantation of 28 premolar donor teeth in 24 orthodontic patients. Angle Orthod 2008;78(1):12-19

16 Yang S, Jung BY, Pang NS. Outcomes of autotransplanted teeth and prognostic factors: a 10-year retrospective study. Clin Oral Investig 2019;23(1):87-98

17 Moorrees CF, Fanning EA, Hunt EE Jr. Age variation of formation stages for ten permanent teeth. J Dent Res 1963;42:1490-1502

18 Tunc SK, Kayasan MS, Ozeroglu E, Eroglu CN. Malpositioned canine treatment with autotransplantation and laser. Eur J Dent 2017;11(3):395-397

19 Murtadha L, Kwok J. Do autotransplanted teeth require elective root canal therapy? A long-term follow-up case series. J Oral Maxillofac Surg 2017;75(9):1817-1826

20 Schätzle M, Tanner SD, Bosshardt DD. Progressive, generalized, apical idiopathic root resorption and hypercementosis. J Periodontol 2005;76(11):2002-2011

21 Tsukiboshi M. Autogenous tooth transplantation: a reevaluation. Int J Periodontics Restorative Dent 1993;13(2): 120-149 